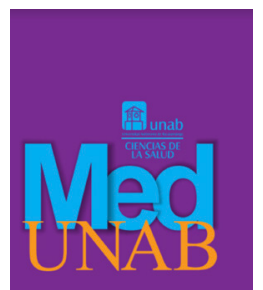

REVISTA DE LA FACULTAD

DE CIENCIAS DE LA SALUD

\title{
Manejo práctico de los nuevos anticoagulantes orales en fibrilación auricular no valvular
}

Practical use of new oral anticoagulants in non-valvular atrial fibrillation

Manejo prático dos novos anticoagulantes orais na fibrilação atrial não valvar

Marlon Adrián Laguado-Nieto, MD., Esp. ${ }^{1}$ (D), Laura Yesenia Ardila-Acuña, $M D .^{2}$ (D), Jairo Alberto Mayorga-Quintero, MD. ${ }^{3}$ (D), Jully Andrea Rangel-Vera, MD. ${ }^{4}$ (D)

1. Médico, especialista medicina interna, docente medicina Universidad de Santander, Bucaramanga, Santander, Colombia.

2. Médica, hospitalaria en la clínica Chicamocha, Bucaramanga, Santander, Colombia.

3. Médico, residente de especialización en medicina interna de la Universidad Autónoma de Bucaramanga, clínica FOSCAL. Floridablanca, Santander, Colombia.

4. Médica, Toronto, Canadá.

Correspondencia: Marlon Adrian Laguado Nieto, Médico Especialista Medicina Interna, Docente Universidad de Santander. Carrera 28 \#33 - 43. Dirección postal: 680002 Bucaramanga. Colombia. E - mail: adriannieto_007@hotmail.com

Cómo citar: Laguado-Nieto MA, Ardila-Acuña LC, Mayorga-Quintero JA, Rangel-Vera JA. Manejo práctico de los nuevos anticoagulantes orales en fibrilación auricular no valvular. MedUNAB. 2019;22(1):38-50 . doi: $10.29375 / 01237047.2823$

\section{INFORMACIÓN ARTÍCULO}

Artículo recibido: 19 de diciembre de 2017

Artículo aceptado: 09 de abril de 2019

DOI: https://doi.org/10.29375/01237047.2823

\section{RESUMEN}

Introducción. La fibrilación auricular es la arritmia más frecuente en la práctica clínica, cuya incidencia viene en ascenso alcanzando el $8 \%$ en edades que superan los 80 años, con un impacto importante en la morbimortalidad relacionado con las complicaciones cerebrovasculares. El objetivo es identificar los aspectos farmacológicos y de manejo práctico de los nuevos anticoagulantes orales en paciente con fibrilación auricular no valvular. 
División de los temas tratados. Se realizó una revisión bibliográfica no sistemática en bases de datos y bibliotecas electrónicas (PubMed, Cochrane, Lilacs) incluyendo artículos desde 2008 hasta 2019, en idioma inglés y español que contuvieran los tópicos de interés. Se realizó una descripción detallada de las generalidades de los anticoagulantes orales en fibrilación auricular, incluyendo dabigatrán, rivaroxabán, apixabán y edoxabán; monitorización de la actividad anticoagulante; descripción del manejo perioperatorio de los nuevos anticoagulantes orales y reversión de la anticoagulación para los nuevos anticoagulantes orales.

Conclusiones. El uso de nuevos anticoagulantes orales en paciente con fibrilación auricular no valvular es una buena alternativa, con facilidad de administración oral, sin aumento significativo del riesgo de sangrado, comparado con warfarina, con la ventaja de no requerir monitoría continua con paraclínicos.

Palabras clave:

Anticoagulantes; Fibrilación Auricular; Dabigatrán; Rivaroxabán; Accidente Cerebrovascular.

\section{ABSTRACT}

Introduction. Atrial fibrillation is the most common arrhythmia in clinical practice. Its incidence has been on the rise, reaching $8 \%$ among those patients over the age of 80 , with a significant impact on morbimortality related to cerebrovascular complications. The objective is to identify aspects of pharmacology and practical use of the new oral anticoagulants in patients with non-valvular atrial fibrillation.

Division of topics covered. A non-systematic literature research was conducted in databases and digital libraries (PubMed, Cochrane, Lilacs), including articles from 2008 to 2019, both in English and in Spanish that included the topics of interest. A detailed description of the generalities of oral anticoagulants for atrial fibrillation was made, including dabigatran, rivaroxaban, apixaban and edoxaban; monitoring anticoagulant activity; description of the perioperative use of new oral anticoagulants and reversal of anticoagulation for the new oral anticoagulants.

Conclusions. The use of new oral anticoagulants in patients with non-valvular atrial fibrillation is a good alternative, with easy oral administration, without significant increase in the risk of bleeding, compared to warfarin, and with the advantage of not requiring continuous paraclinical monitoring.

Keywords:

Anticoagulants; Atrial Fibrillation; Dabigatran; Rivaroxaban; Cerebrovascular Accident.

\section{RESUMO}

Introdução. A fibrilação atrial é o tipo de arritmia mais frequente na prática clínica, cuja incidência está em ascensão, atingindo $8 \%$ nas idades acima de 80 anos, com impacto importante na morbimortalidade relacionada às complicações cerebrovasculares. O objetivo é identificar os aspectos farmacológicos e de manejo prático dos novos anticoagulantes orais em pacientes com fibrilação atrial não valvar. Divisão dos tópicos abordados. Foi realizada uma revisão bibliográfica não sistemática nas bases de dados e bibliotecas eletrônicas (PubMed, Cochrane, Lilacs), incluindo artigos de 2008 até 2016, em inglês e espanhol, que continham os tópicos de nosso interesse. Foi feita uma descrição detalhada das generalidades dos anticoagulantes orais na fibrilação atrial, incluindo dabigatrana, rivaroxabana, apixabana e edoxabana; monitorização da atividade anticoagulante; descrição do manejo perioperatório dos novos anticoagulantes orais e reversão da anticoagulação para os novos anticoagulantes orais.

Conclusões. $\mathrm{O}$ uso de novos anticoagulantes orais em pacientes com fibrilação atrial não valvar é uma boa alternativa, com facilidade de administração por via oral, 
sem aumento significativo do risco de sangramento, comparado à varfarina, com a vantagem de não necessitar de monitoramento contínuo com paraclínicos.

Palavras-chave:

Anticoagulantes; Fibrilação Atrial; Dabigatrana; Rivaroxabana; Acidente Vascular Cerebral.

\section{Introducción}

La fibrilación auricular (FA) es un problema de salud pública en países como Estados Unidos (1) y, además, es la arritmia cardíaca más frecuente; su prevalencia viene en aumento, debido a una mayor expectativa de vida de la población (2). Los ataques isquémicos transitorios (AIT) y los accidente(s) cerebrovascular(es) (ACV) causados por embolismo son las complicaciones más severas de la fibrilación auricular (3).

La FA se desarrolla cuando las anormalidades estructurales y electrofisiológicas del tejido auricular promueven alteraciones en la formación y propagación del impulso eléctrico, produciendo una activación auricular incoordinada y una contracción auricular no efectiva; esto, junto a un estado protrombótico, promueve la formación de trombos en las aurículas, siendo el sitio más común la auriculilla izquierda $(3,4)$. Lo anterior desarrolla fenómenos embólicos cerebrales (o sistémicos) que aumentan la morbimortalidad en los pacientes. La anticoagulación oral para prevención de fenómenos cardioembólicos se iniciará cuando se tenga un puntaje para la evaluación del riesgo de ACV en fibrilación atrial $($ CHADS2 VASc) $>2(5,6)$ (Tabla 1).

Se estima una prevalencia mayor del $8 \%$ en edades que superan los 80 años, los hombres tienen un riesgo 1.5 veces más alto que las mujeres para desarrollar FA y más del $12 \%$ de los pacientes se encuentra entre los 75 y 84 años, de los cuales un tercio son mayores de 80 años $(1,2)$.

\section{Generalidades de los anticoagulantes orales en fibrilación auricular}

El objetivo del tratamiento con anticoagulantes orales (ACO) en la FA es prevenir la mayoría de los ACV isquémicos y prolongar la vida. El riesgo de ACV sin ACO normalmente excede el riesgo de sangrado, incluso en pacientes de edad avanzada, pacientes con disfunción cognitiva o con frecuentes caídas o

Tabla 1. Escala basada en factores de riesgo y expresada por el sistema de puntuación de la escala CHA2DS2VASc

\begin{tabular}{lll}
\hline C & $\begin{array}{l}\text { Insuficiencia cardíaca congestiva / disfunción ventricular izquierda: } \\
\text { Signos o síntomas de insuficiencia cardíaca o evidencia objetiva de } \\
\text { fracción de eyección del ventrículo izquierdo reducida. }\end{array}$ & 1 \\
\hline H & $\begin{array}{l}\text { Hipertensión: PA en reposo }>140 / 90 \mathrm{mmHg} \text { en al menos dos } \\
\text { ocasiones o tratamiento antihipertensivo actual. }\end{array}$ & 1 \\
\hline A2 & Edad $\geq 75$ & 2 \\
\hline D & $\begin{array}{l}\text { Diabetes mellitus: En ayuno glicemia }>125 \mathrm{mg} / \mathrm{dl} \text { o en tratamiento } \\
\text { con hipoglucemiante oral y/o insulina }\end{array}$ & 1 \\
\hline S2 & ACV / AIT / tromboembolismo & 2 \\
\hline V & Enfermedad vascular: IAM previo, EAOC o placa aórtica & 1 \\
\hline A & Edad $65-74$ & 1 \\
\hline Sc & Ser mujer & 1 \\
\hline
\end{tabular}

Fuente: Tomado y adaptado de: January CT, Wann LS, Alpert JS, Calkins H, Cigarroa JE, Cleveland JC, et al. 2014 AHA / ACC / HRS guideline for the management of patients with atrial fibrillation: A report of the American College of cardiology/American heart association task force on practice guidelines and the heart rhythm society. Circulation. 2014. 
fragilidad. Hay suficiente evidencia en pacientes con CHA2DS2-VASc $\geq 2$ puntos en hombres y $\geq 3$ puntos en las mujeres que se benefician de los ACO (4).

A pesar de la evidencia disponible, sigue siendo frecuente la infrautilización o la interrupción prematura de los ACO. Las complicaciones hemorrágicas, graves y leves, el alto riesgo «percibido» de sangrado con los ACO y los esfuerzos necesarios para monitorizar y ajustar la dosis en la anticoagulación con cumarínicos, son las razones más comunes para interrumpir el tratamiento o no prescribirlos (7).

En 2010, el número estimado de varones y mujeres con FA en todo el mundo era de 20.9 millones y 12.6 millones respectivamente. Se prevé que en 2030 la población de pacientes con FA en la Unión Europea será de 14 a 17 millones, con 120,000-215,000 nuevos diagnósticos cada año. Estas estimaciones indican que la prevalencia de la FA será de aproximadamente el $3 \%$ de los adultos de 20 o más años, con mayor prevalencia en personas mayores y pacientes con hipertensión, insuficiencia cardiaca (IC), enfermedad arterial coronaria (EAC), valvulopatía, obesidad, diabetes mellitus o enfermedad renal crónica (ERC). El aumento de la prevalencia de FA se puede atribuir a una mejor detección de la FA silente, junto con el envejecimiento y otras entidades que favorecen el desarrollo de FA $(3,4)$.

A continuación, se darán a conocer las características, función e indicación de los ACO con el fin de ampliar el conocimiento de estos fármacos y disminuir las limitaciones de su uso (Tabla 3, Tabla 4).

Tabla 3. Características farmacológicas de los nuevos anticoagulantes orales

\begin{tabular}{|c|c|c|c|c|}
\hline Anticoagulante & Dabigatrán* & Rivaroxabán** & Apixabán $¥$ & Edoxaban $\mathbb{S}$ \\
\hline Presentación & $\begin{array}{l}\text { Cap. } 150 \text { mg, } 110 \text { y } \\
75 \mathrm{mg}\end{array}$ & Com. 20, 15 y $10 \mathrm{mg}$ & $2.5-5 \mathrm{mg}$ & \\
\hline Factor inhibido & Trombina & $\mathrm{Xa}$ & $\mathrm{Xa}$ & $\mathrm{Xa}$ \\
\hline Biodisponibilidad & $6 \%$ & $80 \%$ & $60 \%$ & $50 \%$ \\
\hline \multirow{2}{*}{ Dosis } & $150 \mathrm{mg} \mathrm{c} / 12 \mathrm{~h}$ & \multirow[t]{2}{*}{$20 \mathrm{mg} \mathrm{c} / 24 \mathrm{~h}$} & $5 \mathrm{mg} \mathrm{c} / 12 \mathrm{~h}$ & $60 \mathrm{mg} \mathrm{c} / 24 \mathrm{~h}$ \\
\hline & $110 \mathrm{mg} \mathrm{c} / 12 \mathrm{~h}$ & & $2.5 \mathrm{mg} \mathrm{c} / 12 \mathrm{~h}$ & $30 \mathrm{mg} \mathrm{c} / 24 \mathrm{~h}$ \\
\hline $\begin{array}{l}\text { Absorción } \\
\text { con comida }\end{array}$ & Retrasa & Aumenta & No afecta & No reportado \\
\hline $\begin{array}{l}\text { Metabolismo } \\
\text { Hepático } \\
(\text { CYP3A4) }\end{array}$ & No & $\mathrm{Si}$ & $\mathrm{Si}$ & Moderado \\
\hline $\begin{array}{l}\text { Interacciones } \\
\text { Medicamentosas }\end{array}$ & $\begin{array}{l}\text { Antiácidos/ } \\
\text { Amiodarona/ } \\
\text { Verapamilo/ } \\
\text { Ketoconazol/ } \\
\text { Carbamazepina/ } \\
\text { Fenitoína/ } \\
\text { Fenobarbital/ } \\
\text { Rifampicina/ } \\
\text { Ciclosporinas/ } \\
\text { Tacrolimus. }\end{array}$ & $\begin{array}{l}\text { Ketoconazol/ } \\
\text { Inhibidores de la } \\
\text { proteasa del VIH/ } \\
\text { Amiodarona/ } \\
\text { Verapamilo/ } \\
\text { Ketoconazol/ } \\
\text { Ciclosporinas/ } \\
\text { Tacrolimus. }\end{array}$ & $\begin{array}{l}\text { Ketoconazol/ } \\
\text { Ciclosporinas/ } \\
\text { Tacrolimus. }\end{array}$ & $\begin{array}{l}\text { Ketoconazol/ } \\
\text { Diltiazem. }\end{array}$ \\
\hline Función Hepática & $\begin{array}{l}\text { No recomendado con } \\
\text { transaminasas } 2 \text { veces } \\
\text { por encima del límite } \\
\text { superior de lo normal. }\end{array}$ & $\begin{array}{l}\text { No recomendado en } \\
\text { pacientes cirróticos } \\
\text { con coagulopatías. }\end{array}$ & $\begin{array}{l}\text { No recomendado en } \\
\text { pacientes cirróticos con } \\
\text { coagulopatías. }\end{array}$ & \\
\hline Excreción Renal & $80 \%$ & $35 \%$ & $25 \%$ & $50 \%$ \\
\hline
\end{tabular}




\begin{tabular}{|c|c|c|c|c|}
\hline Monitorización & No & No & No & No \\
\hline Vida Media & $12-17 \mathrm{~h}$ & $5-9 h$ & $12 \mathrm{~h}$ & $10-14 \mathrm{~h}$ \\
\hline $\begin{array}{l}\text { Pico máximo de } \\
\text { concentración en } \\
\text { plasma }\end{array}$ & $1 \mathrm{~h}$ & $2-4 h$ & $3-4 h$ & $1-2 h$ \\
\hline $\begin{array}{l}\text { Reacciones } \\
\text { Adversas }\end{array}$ & $\begin{array}{l}\text { Dolor abdominal, } \\
\text { dispepsia, sangrado, } \\
\text { gastritis, edema, } \\
\text { esofagitis, sangrado } \\
\text { GTI o intracraneal, } \\
\text { prurito. }\end{array}$ & $\begin{array}{l}\text { Agranulocitosis, } \\
\text { sangrado, colestasis, } \\
\text { disuria, sangrado } \\
\text { GTI, ictericia, } \\
\text { hepatitis, calambres } \\
\text { musculares, } \\
\text { síndrome de Steven- } \\
\text { Johnson, síncope, } \\
\text { trombocitopenia. }\end{array}$ & $\begin{array}{l}\text { Anemia, Hemorragia, } \\
\text { Náuseas, } \\
\text { Trombocitopenia, } \\
\text { Epistaxis, Aumento de } \\
\text { transaminasas. }\end{array}$ & \\
\hline Contraindicaciones & $\begin{array}{l}\text { Sangrado, Embarazo, } \\
\text { Lactancia, Cirugía }\end{array}$ & $\begin{array}{l}\text { Sangrado, Lactancia, } \\
\text { Coagulopatía, } \\
\text { Enfermedad } \\
\text { Hepática, Sangrado } \\
\text { Gastrointestinal, } \\
\text { Anestesia Espinal }\end{array}$ & $\begin{array}{l}\text { Sangrado activo, } \\
\text { Hepatopatía asociada } \\
\text { o coagulopatía, } \\
\text { Hipersensibilidad, } \\
\text { Embarazo, Lactancia }\end{array}$ & \\
\hline \multirow{4}{*}{$\begin{array}{l}\text { Uso sugerido } \\
\text { de acuerdo a las } \\
\text { características del } \\
\text { cuadro del paciente }\end{array}$} & \multirow{4}{*}{$\begin{array}{l}\text { Alto riesgo } \\
\text { de accidente } \\
\text { cerebrovascular } \\
\text { (Alta puntuación } \\
\text { CHADS-VASC), } \\
\text { Alto riesgo } \\
\text { de sangrado, } \\
\text { Hemorragias } \\
\text { potencialmente } \\
\text { mortales }\end{array}$} & \multirow{4}{*}{ Ictus Previo } & $\begin{array}{l}\text { Alto riesgo de } \\
\text { sangrado, Hemorragias } \\
\text { potencialmente } \\
\text { mortales. }\end{array}$ & \\
\hline & & & \multirow{3}{*}{$\begin{array}{l}\text { Sangrado } \\
\text { gastrointestinal, } \\
\text { Edad avanzada } \\
\text { (mayores de } 80 \text { años) y } \\
\text { alteración } \\
\text { Funcional renal. }\end{array}$} & \\
\hline & & & & \\
\hline & & & & \\
\hline
\end{tabular}

Estudios: * RE-LY; ** ROCKET-AF; ¥ ARISTOTLE; § ENGAGE AF-TIMI 48.

Fuente: Tomado y adaptado de: Kirchhof P, Benussi S, Kotecha D, Ahlsson A, Atar D, Casadei B, et al. 2016 ESC Guidelines for the management of atrial fibrillation developed in collaboration with EACTS. European Heart Journal. 2016.

\section{Dabigatrán}

Es un inhibidor directo de la trombina y el único de este tipo que se administra oralmente (argatrobán y bivalirudina se administran parenteralmente) (7). Fue el primero de los nuevos anticoagulantes orales (NACO) aprobado en varios países a nivel mundial para prevención de trombos en pacientes con FA. Dabigatrán etexilato es una prodroga que se hidroliza completamente en su forma activa dabigatrán, por esterasas no específicas en el intestino, plasma e hígado. Se puede administrar con o sin alimentos, alcanza su pico a las 2 - 3 horas posteriores a su administración y su vida media es de $12-17$ horas con eliminación predominantemente renal $(80 \%)(8,9)$.
El medicamento es sustrato para el transportador P-glicoproteína (P-gp) y los medicamentos que afectan este sistema alteran su farmacocinética. Los medicamentos que no deberían ser administrados ya que son potentes inhibidores de la (P-gp) son la ciclosporina, itraconazol, ketoconazol sistémico, tacrolimus, rifampicina y dronedarona; tampoco se recomienda la coadministración de medicamentos que son inductores de la (P-gp) como la fenitoína, carbamazepina, rifampicina o los inhibidores de la proteasa (8), además de fármacos antihipertensivos como el verapamilo.

El estudio RELY, aleatorizado y multicéntrico (35), incluyó a 18,113 pacientes con FA y riesgo de ACV que 
Tabla 4. Administración de nuevos anticoagulantes orales y su dosificación según tasa de filtración glomerular

\begin{tabular}{lllll}
\hline Dosis por FDA & Dabigatrán & Rivaroxabán & Apixabán* & Edoxaban \\
\hline TFG $>50 \mathrm{ml} / \mathrm{min}$ & $\begin{array}{l}150 \mathrm{mg} \text { cada } 12 \\
\text { horas }\end{array}$ & $20 \mathrm{mg}$ día & $5 \mathrm{mg}$ cada 12 horas & $60 \mathrm{mg}$ día \\
\hline $\begin{array}{l}\text { TFG } 30-50 \mathrm{~mL} / \\
\text { min }\end{array}$ & $\begin{array}{l}150 \mathrm{mg} \text { cada } 12 \\
\text { horas }\end{array}$ & $15 \mathrm{mg}$ día & $5 \mathrm{mg}$ cada 12 horas & $30 \mathrm{mg}$ día \\
\hline $\begin{array}{l}\text { TFG } 15-30 \mathrm{ml} / \\
\text { min }\end{array}$ & $\begin{array}{l}75 \mathrm{mg} \text { cada } 12 \\
\text { horas }\end{array}$ & $15 \mathrm{mg}$ día & $5 \mathrm{mg}$ cada 12 horas & $30 \mathrm{mg}$ día \\
\hline TFG $<15 \mathrm{ml} / \mathrm{min}$ & No dato & No recomendado & & \\
\hline
\end{tabular}

TFG: Tasa de filtración glomerular. * Dosis $2.5 \mathrm{mg}$ si reúnen 2 o más de los siguientes criterios: edad $\geq 80$ años, peso corporal $<60 \mathrm{~kg}$ o creatinina sérica $\geq 1.5 \mathrm{mg} / \mathrm{dl}$.

Fuente: Tomado y adaptado de: Heidbuchel H, Verhamme P, Alings M, Antz M, Diener HC, Hacke W, et al. Updated European Heart Rhythm Association Practical Guide on the use of non-Vitamin K antagonist anticoagulants in patients with non-valvular atrial fibrillation. Europace. 2015.

tenían uno o más de los siguientes factores de riesgo: falla cardíaca sintomática o fracción de eyección del ventrículo izquierdo (FEVI) $<40 \%$; edad mayor o igual a 75 años o entre los 65 y 74 años, con un factor de riesgo adicional de diabetes mellitus (DM); hipertensión (HTA) o enfermedad arterial coronaria; asignados a recibir dabigatrán $110 \mathrm{mg} 2$ veces al día, dabigatrán $150 \mathrm{mg} 2$ veces al día o warfarina.

El estudio tuvo una duración media de dos años y $\mathrm{su}$ desenlace principal fue un desarrollo de ACV o embolismo sistémico. Se excluyeron pacientes con enfermedad valvular cardíaca severa, depuración de creatinina $(\mathrm{ClCr})<30 \mathrm{ml} / \mathrm{min}, \mathrm{ACV}$ dentro de 14 días, embarazo y enfermedad hepática activa.

Los resultados demostraron que el dabigatrán en dosis de $110 \mathrm{mg} 2$ veces al día, comparado con la warfarina, se asoció con tasas de ACV y embolismo sistémico que fueron similares, así como tasas más bajas de hemorragia mayor. Por otro lado, el dabigatrán en dosis de $150 \mathrm{mg} 2$ veces al día, comparado con la warfarina, presentó tasas más bajas de ACV y embolismo sistémico, pero tasas similares de hemorragia mayor (10).

La dosis usual del dabigatrán es $150 \mathrm{mg} 2$ veces al día (aprobado por la administración de drogas y alimentos FDA y la Agencia Europea de medicamentos EMA). La dosis de $110 \mathrm{mg} 2$ veces al día (solo aprobada por EMA) se recomienda en pacientes mayores a 80 años, que estén en tratamiento concomitante con verapamilo o con dos o más de los siguientes parámetros de alto riesgo de sangrado: edad $75-80$ años, peso $<60 \mathrm{Kg}$, historia de sangrado digestivo, $\mathrm{ClCr} 30-49 \mathrm{ml} / \mathrm{min}$, escala HAS BLED (escala de clasificación del riesgo de sangrado) $\geq 3$, trombocitopenia, uso simultáneo con anti plaquetarios, AINES o esteroides (11).

\section{Rivaroxabán}

Es un inhibidor del Factor Xa, se administra una vez al día alcanzando su efecto pico a las 2-3 horas, con una vida media de 7 - 11 horas; $30 \%$ del medicamento permanece sin cambio cuando se excreta por los riñones, mientras que el resto es metabolizado a nivel hepático por el sistema CYP3A4 $(9,12)$. También es un sustrato para la P-gp; administrar medicamentos que son fuertes inhibidores de la P-gp y CYP3A4 como el ketoconazol o ritonavir, que están contraindicados ya que pueden incrementar la concentración sérica de rivaroxabán y el riesgo de sangrado. Se debe hacer un balance riesgobeneficio para administrar este medicamento en pacientes con alteración en la función renal con inhibidores de la P-gp y CYP3A4 (amiodarona, diltiazem, verapamilo, quinidina, eritromicina, azitromicina).

Por otro lado, la concentración del rivaroxabán se puede reducir con inductores de la P-gp y CYP3A4 como rifampicina, fenitoína o carbamazepina (pueden presentarse efectos sobre los niveles plasmáticos) $(14,15)$. Se contraindica su uso en pacientes con enfermedad hepática activa Child pugh B-C $(11,12)$. 
El estudio ROCKET AF fue un ensayo multicéntrico doble ciego aleatorizado, con 14,264 pacientes con FA no valvular, asignados a recibir rivaroxabán $20 \mathrm{mg}$ al día por vía oral (VO) $(15 \mathrm{mg}$ para pacientes con $\mathrm{ClCr}$ $30-49 \mathrm{ml} / \mathrm{min}$ ) o warfarina. El objetivo principal fue determinar si el rivaroxabán era o no inferior a la warfarina para el objetivo principal de ictus de cualquier causa o embolia sistémica en la población tratada.

Se incluyeron pacientes con antecedente de AIT, ACV o embolismo sistémico, o al menos 2 de los siguientes: edad $>75$ años, falla cardíaca o FEVI $<35 \%$, HTA o DM. El rivaroxabán no fue inferior a la warfarina desde el punto final primario de ACV o embolismo sistémico, con una tasa anual de $2.12 \%$ vs $2.42 \%$ respectivamente $(\mathrm{P}$ $<0.001$ para no inferioridad, $\mathrm{P}=0.12$ para superioridad). La tasa de ACV isquémico fue similar entre los grupos de rivaroxabán y warfarina $(1.34 \%$ vs $1.42 \%$ por año respectivamente, $\mathrm{P}=0.581$ ) y el $\mathrm{ACV}$ hemorrágico ocurrió con menos frecuencia en el grupo del rivaroxabán $(0.26 \%$ vs $0.44 \%$ por año, $\mathrm{P}=0.024)(14,15)$.

Elrivaroxabán no fue inferior a la warfarina en la prevención de ictus embólico o embolia sistémica, ni aumentó de manera significativa las hemorragias graves o clínicamente relevantes; sin embargo, redujo significativamente las hemorragias intracraneales, de órgano crítico y mortales en relación con la warfarina $(16,17)$.

\section{Apixabán}

Es otro NACO que ejerce una actividad anticoagulante similar al rivaroxabán, inhibe de manera directa el factor Xa que se forma por las dos vías de la coagulación intrínseca y extrínseca, se absorbe rápidamente tras la administración oral, su biodisponibilidad es de aproximadamente $50 \%$ (24). Tiene una vida media de 8 - 15 horas. El aclaramiento renal de apixabán es diverso (metabolismo, excreción biliar y excreción directa en el intestino), una contribución menor de CYP3A4 genera que las interacciones delCYP3A4 sean menos importantes para este medicamento. Al igual que con otros NACO, no se debería administrar concomitante con ketoconazol, itraconazol, ritonavir o claritromicina (19).

En 2012, se convirtió en el segundo inhibidor del factor Xa en ser aprobado para pacientes con FA no valvular, se demostró su seguridad y efectividad en dos grandes ensayos de pacientes con FA o valvular:

a) El estudio AVERROES (apixabán versus ácido acetilsalicílico), para prevenir el accidente cerebrovascular $(\mathrm{ACV})$ en pacientes con fibrilación auricular, que no son candidatos a anticoagulación con warfarina. Se presentó dificultad para realizar la prueba de INR $(43 \%)$ e incapacidad para mantener INR en rango terapéutico (42\%). Fueron asignados al azar y a ciegas ácido acetilsalicílico diaria o apixabán dos veces al día $(5 \mathrm{mg}$ ) en cada uno de los grupos; el objetivo de eficacia (ictus o embolia sistémica) se observó en el $1.6 \%$ por año en el grupo de apixabán y el $3.7 \%$ por año en el grupo de ácido acetilsalicílico (Hazard ratio $=0.45$; IC $95 \%$, $0.32-0.62 ; p<0.001)$. El sangrado se observó en el 1.4 $\%$ por año en el grupo de apixabán y el $1.2 \%$ por año en el grupo de ácido acetilsalicílico (Hazard ratio 1.13; IC $95 \%, 0.74-1.75 ; p=0.57)$. El estudio fue suspendido prematuramente al observarse clara superioridad del apixabán, que mostraba menos eventos tromboembólicos sin incremento del riesgo de sangrado, comparados con el grupo de ácido acetilsalicílico $(16,18,19)$.

b) El estudio ARISTOTLE, aleatorizado y doble ciego, evidencia la reducción de accidente cerebro vascular en contexto de FA $(18,21)$. Se realizó comparación de apixabán (dosis de $5 \mathrm{mg} /$ día) con warfarina (objetivo de INR entre 2 y 3 ) en 18,201 pacientes con fibrilación auricular y, al menos, otro factor de riesgo cardiovascular para ictus. La variable principal fue el ictus isquémico o hemorrágico o la embolia sistémica $(15,18,20,21,22)$. El apixabán fue superior a la warfarina respecto a la prevención del riesgo de ictus o embolia sistémica, con disminución en el riesgo de sangrado y muerte (19).

Se concluyó que el apixabán fue superior a la warfarina en prevenir ictus o embolias sistémicas, causó menos sangrados y derivó en menor mortalidad. Los pacientes del grupo warfarina mantuvieron un INR de 2 - 3 solo durante el $66 \%$ del tiempo de seguimiento, la mejoría en el evento primario de eficacia fue derivado de la reducción de los ictus hemorrágicos, mientras que los eventos isquémicos (ictus isquémico y embolias sistémicas) fueron similares en ambos grupos; por lo tanto, en prevención antitrombótica, el apixabán parece similar a la warfarina, mientras que la reducción de los ictus hemorrágicos probablemente esté en relación con la disminución de los sangrados que se refleja en el evento primario de seguridad (19).

La dosis de apixabán es de $5 \mathrm{mg}$ vía oral cada 12 horas; sin embargo, en pacientes mayores de 80 años, peso $\leq$ a 60 $\mathrm{kg}$ y creatinina sérica mayor igual de $133 \mathrm{micromol} / \mathrm{L}$, se administra dosis de $2.5 \mathrm{mg}$ cada $12 \mathrm{~h}$. Está contraindicado en $\mathrm{CrCl}$ menor de $25 \mathrm{Ml} / \mathrm{min}$ o en diálisis (22). 


\section{Edoxabán}

Es un inhibidor oral directo, específico de Factor Xa con una selectividad aproximada de 10,000 veces por Xa sobre la trombina. Fue aprobado por la FDA en enero de 2015 para la prevención de accidente cerebrovascular, se absorbe rápidamente y se estimó que su biodisponibilidad absoluta es del $58.3 \%$. Tiene mecanismos duales de eliminación, aproximadamente un tercio se elimina a través de los riñones y el resto por las heces (16).

En el ensayo clínico ENGAGE AF-TIMI 48, un estudio internacional aleatorizado y doble ciego, se compara la eficacia y seguridad de dos dosis de edoxabán $(30$ y $60 \mathrm{mg}$ al día) con warfarina, en 20,500 pacientes con FA y un riesgo moderado-alto de ACV en 24 meses. La tasa de ictus isquémico fue similar a dosis altas en edoxabán y warfarina, pero fue mayor con el régimen de edoxabán a dosis baja.

La incidencia de ictus o embolia sistémica en el grupo de warfarina fue de $1.50 \%$ anual, comparada con un 1.18 $\%$ con la dosis alta de edoxabán y $1.61 \%$ con la dosis baja. Los sangrados mayores se produjeron en un 3.43 $\%$ con el grupo de warfarina, versus $2.75 \%$ con la dosis elevada de edoxabán y un $1.61 \%$ con la dosis baja.

Además, la tasa anual de muertes de causa cardiovascular fue del $3.17 \%$ versus $2.74 \%$ y $2.71 \%$ para warfarina, dosis elevada y dosis baja de edoxabán, respectivamente. En cuanto al evento combinado de ictus, embolia sistémica y muerte por causa cardiovascular, las tasas fueron del $4.43 \%$ con warfarina y $3.85 \%$ con dosis elevada de edoxabán (22).

\section{Monitorización de la actividad anticoagulante}

\section{Inhibidores directos de la trombina}

A pesar de que no hay necesidad de monitorización rutinaria del dabigatrán en pacientes con FA, hay ciertas situaciones de excepción como las cirugías de urgencias, donde el incremento en las concentraciones séricas aumenta el riesgo de sangrado. Se evidencia prolongación del TPT a más que del TP $(22,23)$.

El tiempo de coagulación de ecarina (ECT por sus siglas en inglés) provee una medida directa de la actividad de los inhibidores directos de la trombina (IDT). Existen test calibrados para dabigatrán disponibles como el análisis cromogénico, que proveen de una correlación lineal con las concentraciones de dabigatrán. Los valores prolongados de ECT ( $>3$ veces) indican alto riesgo de sangrado (22).

El tiempo de trombina (TT) es muy sensible ante la presencia de dabigatrán y valores normales excluyen valores muy bajos del medicamento $(12,22)$. El tiempo de trombina diluido (dTT) predice de forma más precisa la actividad anticoagulante de dabigatrán. Se evidencia una relación lineal con las concentraciones de dabigatrán y es más adecuado para evaluar cuantitativamente su concentración.

Valores de dTT $>65 \mathrm{~s}$ indican concentraciones de dabigatrán $>200 \mathrm{ng} / \mathrm{ml}$ con alto riesgo de sangrado y obligan a ajustes en dosis de pacientes con factores de riesgo. Estudios donde comparan TT y TPTa en monitorización de pacientes que toman dabigatrán han encontrado que el TT es muy sensible ante la presencia del medicamento, y que el TPTa es útil como test cualitativo, pero tanto TT como TPTa tienen correlación moderada con los niveles en sangre (17).

\section{Inhibidores Factor Xa}

\section{Rivaroxabán}

El TP tiene una correlación lineal con las concentraciones del medicamento en el plasma. Valores normales indican no actividad anticoagulante. Valores dos veces por encima de lo normal pueden indicar concentraciones de $700 \mathrm{mcgv} / \mathrm{ml}$, aunque esto varía dependiendo el tipo de reactivo que se utilice en el laboratorio (neoplastina plus es más sensible que neoplastina). El INR no debe ser usado para monitorización $(22,23)$.

\section{Edoxabán y apixabán}

El TP no puede ser usado para evaluar su efecto anticoagulante, para esta clase de anticoagulantes el análisis (cuantitativo) cromogénico Anti-factor Xa es un método válido para evaluar concentraciones plasmáticas del medicamento, tiene disponibilidad limitada, al igual que ECT y dTT que evalúan solamente al dabigatrán $(12,22,23)$.

\section{Manejo perioperatorio de los nuevos anticoagulantes orales}

Una situación relativamente frecuente en la actividad clínica es el manejo perioperatorio de pacientes que están en manejo con NACO. Durante los ensayos clínicos de estos medicamentos se presentaron interrupciones en la continuidad del medicamento en un $25 \%$ en el RE-LY, $33 \%$ en el ROCKET - AF y $34 \%$ en el ARISTOTLE 
Tabla 2. Clasificación de intervenciones quirúrgicas electivas según el riesgo de sangrado

\begin{tabular}{ll}
\hline Administración de NOAC & Intervención \\
\hline $\begin{array}{l}\text { Intervenciones que no } \\
\text { requieren de suspensión } \\
\text { de NOAC }\end{array}$ & Intervenciones Dentales. \\
\hline & Oftalmológicas: Intervención para cataratas o Glaucoma. \\
\hline & Cirugía endoscópica. \\
\hline & $\begin{array}{l}\text { Cirugía Superficial: incisión de abscesos, escisiones } \\
\text { dermatológicas, entre otras. }\end{array}$ \\
\hline $\begin{array}{l}\text { Intervenciones con menor } \\
\text { riesgo de hemorragia }\end{array}$ & Endoscopia más biopsia. \\
\hline & Biopsia de Próstata o vejiga. \\
\hline $\begin{array}{l}\text { Estudio electrofisiológico o ablación derecha para taquicardia } \\
\text { supra ventricular. }\end{array}$ \\
\hline $\begin{array}{l}\text { Intervenciones con mayor } \\
\text { y un mayor riesgo } \\
\text { tromboembólico }\end{array}$ & $\begin{array}{l}\text { Angiografía coronaria, marcapasos o implante de CDI. } \\
\text { riesgo de hemorragia }\end{array}$ \\
\hline $\begin{array}{l}\text { VT. } \\
\text { Ablación de complón con catéter para taquicardia supra ventricular sencilla, }\end{array}$ \\
\hline Anestesia epidural, punción lumbar diagnóstica. \\
\hline & Cirugía torácica, cirugía abdominal. \\
\hline & Resección transuretral de próstata, biopsia renal o hepática. \\
\hline
\end{tabular}

Fuente: Tomado y adaptado de: Heidbuchel H, Verhamme P, Alings M, Antz M, Diener HC, Hacke W, et al. Updated European Heart Rhythm Association Practical Guide on the use of non-Vitamin K antagonist anticoagulants in patients with non-valvular atrial fibrillation. Europace. 2015

(24). Es importante clasificar el tipo de intervención quirúrgica que se le realizará al paciente, ya que hay procedimientos de alto a bajo riesgo de sangrado (Tabla 2), así como conocer la tasa filtración glomerular.

Para pacientes que están tomando rivaroxabán, apixabán y edoxabán, que se someten a intervenciones quirúrgicas de bajo riesgo, con depuración de creatinina de 30 a más de $80 \mathrm{ml} / \mathrm{min}$, la última dosis de estos medicamentos deberá ser $>$ a 24 horas antes de la cirugía; si la depuración de creatinina es de 15 a $30 \mathrm{ml}$ / min, la última dosis deberá ser $>$ a 36 horas; y si la intervención quirúrgica es de alto riesgo, la última dosis deberá ser $>$ a 48 horas antes de la cirugía $(22,25)$.

Con respecto al dabigatrán, si se trata de una cirugía de bajo riesgo y la depuración de creatinina es $>80 \mathrm{ml} /$ min, la última dosis del anticoagulante deberá ser $>24$ 
horas previas al procedimiento; $\mathrm{ClCr} 50-80 \mathrm{ml} / \mathrm{min}$, el tiempo es de $>36$ horas; y ClCr $30-50 \mathrm{ml} / \mathrm{min}$, es $>48$ horas. Para procedimientos de alto riesgo con $\mathrm{ClCr}$ $>80 \mathrm{ml} / \mathrm{min}$, se deberá suspender por $>48$ horas; $\mathrm{ClCr}$ $50-80 \mathrm{ml} / \mathrm{min}$, se suman 24 horas más a la última dosis del medicamento ( $>72$ horas); y depuraciones 30 - $50 \mathrm{ml} / \mathrm{min}$, se suman otras 24 horas (>96 horas). No se indica con $\mathrm{ClCr}<30 / \mathrm{ml}(22,25)$.

Después de la intervención quirúrgica, ¿cuándo se reinician los NACO?; hay que tener presente nuevamente el riesgo de sangrado del procedimiento (alto / bajo), el control del sangrado, la hemostasia y otros factores como si el paciente está inmovilizado por la cirugía o si presenta íleo posquirúrgico, lo cual altera la absorción de los anticoagulantes (26).

\section{Reversión de la anticoagulación para los nuevos anticoagulantes orales}

Cuando se emplean NACO en la práctica clínica, existen riesgos de sangrados, si bien en menos proporción que con uso de warfarina en sangrados intracraneales, no hay diferencias significativas en otros sangrados fatales (27). Por lo anterior, es importante revertir la anticoagulación sobre todo ante hemorragias potencialmente mortales, traumas severos y requerimiento de procedimientos quirúrgicos de urgencias en pacientes anticoagulados. Otras medidas, como transfusiones, manejo local, lavados gástricos, carbón activado y diálisis (para dabigatrán), pueden ser utilizadas en sangrados leves a moderados.

El desarrollo de agentes de reversión de la anticoagulación tiene varios desafíos: poder generar un impacto positivo en el resultado global (evitar sangrados, reversión rápida, acción sostenida, etc.), evitar estados protrombóticos, generar bajos efectos adversos y que sean costo-efectivos (28). A continuación se describen tres compuestos actualmente en desarrollo.

\section{Idarucizumab}

Es un fragmento de anticuerpo monoclonal humanizado Fab, que se dirige específicamente contra el dabigatrán. Se une a dabigatrán con una afinidad 350 veces más potente que la afinidad de unión del dabigatrán a la trombina. Demostró invertir la actividad del dabigatrán pocos minutos después de la infusión de dos dosis de 2.5 gr en pacientes con FA anticoagulados con dabigatrán, con hemorragia mayor o cirugía urgente. Demostró normalizar el dTT y ECT, mantener valores normales durante las siguientes 24 horas, hemostasia intraoperatoria entre la mayoría de los pacientes llevados a cirugía y restauración de hemostasia con una media de 11.4 horas, en pacientes con hemorragia grave. Cinco pacientes con eventos trombóticos (5.5\%) fueron reportados. En octubre de 2015, idarucizumab recibió aprobación de la FDA con indicaciones para la reversión de dabigatrán en pacientes que requieren cirugía de urgencia o hemorragia mayor $(29,30)$.

En el estudio REVERSE AD se reclutaron 503 pacientes con fibrilación auricular en tratamiento con dabigatrán, se aleatorizaron a los sujetos en dos grupos (grupo A, con 301 pacientes con sangrado digestivo o sangrado intracraneal; y grupo B, con 202 pacientes con indicación de cirugía de urgencia), y se les administró idarucizumab 5 gramos (2 dosis de 2.5 gramos administradas con una diferencia de no más de 15 minutos). El objetivo primario fue el porcentaje máximo de reversión del efecto anticoagulante del dabigatrán y los objetivos secundarios incluyeron la restauración de la hemostasia y demás medidas de seguridad.

El porcentaje de reversión del efecto del dabigatrán, medido por el tiempo de trombina diluido, fue del 100 $\%$ en ambos grupos. El tiempo medio de control de sangrado en pacientes con hemorragia intracraneal fue de 11.4 horas y del sangrado digestivo de 3.5 horas. En el grupo B la media del tiempo para poder ser llevado a procedimiento quirúrgico de urgencia fue de 1.6 horas.

La tasa de eventos trombóticos fue del $4.8 \%$ y $6.8 \%$ a los 30 y 90 días respectivamente, similar en ambos grupos. Estos episodios se presentaron 72 horas después de la administración del medicamento, sin embargo, es más probable que esto refleje un estado protrombótico de base que un efecto directo del medicamento, ya que este no tiene actividad procoagulante. Una limitación del estudio fue no disponer de grupos de control (32).

\section{Andexanet Alfa}

Se trata de una proteína recombinante del factor Xa humano modificada, que no tiene actividad coagulante, pero conserva su capacidad para unirse a inhibidores del factor Xa. En recientes estudios clínicos, se logró demostrar la reversión del efecto de rivaroxabán y apixabán (30), mediante inyección en bolo seguida de infusión de dos horas de andexanet. La inhibición del factor Xa se redujo y la generación de trombina se restauró significativamente vs placebo $(\mathrm{P}<0.001)$, fue bien tolerado sin documentación de efectos adversos (31). Si bien no se han presentado eventos trombóticos, se ha documentado un leve perfil protrombótico por reducción en el factor tisular inhibidor que pudiera llegar a generar complicaciones en pacientes predispuestos. 
En el estudio ANNEXA-4 se analizaron 352 pacientes que estaban tomando rivaroxabán o apixabán, quienes presentaban sangrado agudo mayor dentro de las 18 horas posteriores de haberse administrado el inhibidor del factor Xa. El objetivo primario fue evaluar el porcentaje de actividad anti factor Xa después de administrar andexanet (una modificación recombinante inactiva del factor Xa), y el porcentaje de hemostasia a las 12 horas después de la infusión del medicamento.

En los pacientes que recibieron andexanet la disminución media de actividad anti factor Xa fue del 92 $\%$ en pacientes que recibieron apixabán y rivaroxabán. La hemostasia se logró en el $82 \%$ de los pacientes. La mortalidad fue del $14 \%$ a los 30 días y la tasa de eventos trombóticos fue del $10 \%$, relacionados estos últimos con la suspensión o la demora en el reinicio de la anticoagulación. Una vez reiniciado el manejo anticoagulante no se documentaron eventos trombóticos durante el seguimiento a los 30 días (33).

\section{Ciraparantag}

Es un pequeño compuesto sintetizado originalmente como agente inhibidor de las heparinas, que ha demostrado revertir el efecto de los NACOS (dabigatrán, rivaroxabán, apixabán, edoxabán), impidiendo la unión de dichos fármacos con su receptor (2). Estudios en humanos encontraron corrección de los tiempos de coagulación tras administración de $100 \mathrm{mg}$ de ciraparantag; posterior a uso de $60 \mathrm{mg}$ de edoxabán, se mantiene su efecto 24 horas después sin evidencia de efectos protrombóticos ni otros efectos adversos (31). Un desafío que enfrenta este fármaco es la medición de su eficacia, debido a que se une, en grados variables, a la mayoría de los reactivos utilizados para tal fin, dando pruebas de coagulación alteradas. Faltan estudios clínicos significativos para la aprobación de su uso (32).

\section{Conclusiones}

Lafibrilación auricular es una enfermedad cuya prevalencia va en ascenso a medida que la población envejece, está asociada a diversos factores de riesgo cardiovasculares tales como la diabetes y la hipertensión. Es una causa importante de enfermedad cerebrovascular, derivada de la formación de trombos y embolia sistémica desde las aurículas, que condicionan a una gran mortalidad y morbilidad; esta última dada por las secuelas neurológicas que aumentan los costos en cuidados en salud.

Para prevenir la formación de trombos y la embolia sistémica, es necesario la anticoagulación: en caso de fibrilación auricular de origen no valvular se puede emplear la warfarina o los nuevos anticoagulantes orales (dabigatrán, rivaroxabán, apixabán) por su adecuado perfil de seguridad, sin aumento significativo de sangrado mayor y sin necesidad de control periódico con laboratorio, en comparación con la warfarina.

Es necesario tener presentes varios aspectos con el uso de nuevos anticoagulantes orales, como la tasa de filtración glomerular del paciente, pues se deberán ajustar las dosis y en algunos casos suspender el medicamento; asimismo, se debe tener en cuenta la la insuficiencia renal estadio V o la hemodiálisis. También se deben tener presentes las posibles interacciones entre medicamentos que pueden aumentar su concentración en sangre, con aumento del riesgo de sangrado. En caso de requerir cirugía es importante reconocer el riesgo de sangrado y suspender previamente el medicamento según comorbilidades y el tipo de NACO que se esté utilizando, además revertir en caso de ser emergente.

\section{Conflicto de intereses}

Los autores declaran no tener conflictos de interés.

\section{Referencias}

1. Deshpande S, Catanzaro J, Wann S. Atrial fibrillation prevalence and scope of the problem. Cardiac Electrophysiology Clinics. 2014; 6 (1):1 - 4. doi: 10.1016/j. ccep.2013.10.006

2. Renoux C, Patenaude V, Suissa S. Incidence, mortality, and sex differences of non-valvular atrial fibrillation: A population-based study. Journal of the American Heart Association. 2014; 3 (6):1 - 8. doi: 10.1161/ JAHA.114.001402

3. Mulpuru S, Rabinstein A, Asirvatham S. Atrial fibrillation and stroke a neurologic perspective. Cardiac Electrophysiology Clinics 2014; 6(1):31 - 41. doi: 10.1016/j.ccep.2013.11.001

4. Castellano JM, Chinitz J, Willner J, Fuster V. Mechanisms of stroke in atrial fibrillation. Cardiac Electrophysiology Clinics. 2014; 6(1):5 - 15. doi: 10.1016/j. ccep.2013.10.007.

5. January CT, Wann LS, Alpert JS, Calkins H, Cigarroa JE, Cleveland JC, et al. 2014 AHA/ACC/HRS Guideline for the management of patients with atrial fibrillation: A report of the American College of cardiology/American heart association task force on practice guidelines and the heart rhythm society. Circulation. 2014; 130(23)201 -267. doi: 10.1161/ CIR.0000000000000041 
6. Kirchhof P, Benussi S, Kotecha D, Ahlsson A, Atar D, Casadei B, et al. 2016 ESC Guidelines for the management of atrial fibrillation developed in collaboration with EACTS. European Heart Journal. 2016; 37(38):2893 - 2962. doi: 10.1093/eurheartj/ehw210

7. Harter K, Levine M, Henderson S. O. Anticoagulation drug therapy: a review. The Western Journal of Emergency Medicine. 2015; 16(1):11 - 7. doi: 10.5811/ westjem.2014.12.22933

8. Tatjana S, Gregory Y. Novel oral anticoagulants in non-valvular atrial fibrillation. Cardiovascular. Hematological Agents in Medicinal Chemistry. 2014;26 (2) :115 - 129. doi: 10.1016/j.beha.2013.07.008

9. Cove C, Hylek E. An updated review of target-specific oral anticoagulants used in stroke prevention in atrial fibrillation, venous thromboembolic disease, and acute coronary syndromes. Journal of the American Heart Association. 2013; 2(5):115 - 219. doi: 10.1161/ JAHA.113.000136

10. Connolly S, Ezekowitz M, Yusuf S, Eikelboom J, Oldgren J, Parekh A, et al. Dabigatran versus warfarin in patients with atrial fibrillation. The New England Journal of Medicine. 2009; 361(12):1139 - 1151. doi: 10.1056/NEJMoa0905561

11. Hinojar R, Jiménez-Natcher J, Fernández-Golfín C, Zamorano J. New oral anticoagulants: a practical guide for physicians. European Heart Journal - Cardiovascular Pharmacotherapy. 2015; 1(2):134 - 145. doi: 10.1093/ehjcvp/pvv002

12. Ferns S, Naccarelli G. New Oral Anticoagulants. Their Role in Stroke Prevention in High-Risk Patients with Atrial Fibrillation. Medical Clinics of North America. 2015; 99(4):759 - 80. doi: 10.1016/j. mona.2015.02.006

13. Shafeeq H, Tran T. New oral anticoagulants for atrial fibrillation: are they worth the risk? P\&T : A Peer-Reviewed Journal for Formulary Management. [Internet].2014.[citado el 03 de Junio del 2019]; 39(1):54 - 64. Recuperado a partir de: https://www.ncbi.nlm. nih.gov/pmc/articles/PMC3956385/

14. Patel MR, Mahaffey KW, Garg J, Pan G, Singer DE, Hacke W, et al. Rivaroxaban versus warfarin in nonvalvular atrial fibrillation. The New England Journal of Medicine. 2011; 365(10):883-91. doi: 10.1056/NEJMoa1009638

15. López-Sendón J, Merino J. Resultados e implicaciones del estudio ROCKET. Revista Española de Cardiología Suplementos. 2013; 13(C):42 - 46. doi: 10.1016/S1131-3587(13)70058-X

16. Mekaj Y, Mekaj A, Duci S, Miftari E. New oral anticoagulants: Their advantages and disadvantages compared with vitamin $\mathrm{K}$ antagonists in the prevention and treatment of patients with thromboembolic events. Therapeutics and Clinical Risk Management.
2015(11):967 - 977. doi: 10.2147/TCRM.S84210

17. Shamoun F, Obeid H, Ramakrishna H. Novel anticoagulants in atrial fibrillation: Monitoring, reversal and perioperative management. BioMed Research International. 2015; 424031. doi: 10.1155/2015/424031

18. Steinberg B, Piccini, J. Anticoagulation in atrial fibrillation. British Medical Journal. 2014; 14(348) 2116. doi: 10.1136/bmj.g2116

19. Martínez-Rubio A, Martínez-Torrecilla R. Evidencias actuales de los nuevos anticoagulantes orales en el tratamiento de la fibrilación auricular no valvular: comparación de subestudios. Revista Espanola de Cardiologia. 2015; 68(3):185 - 189. doi: 10.1016/j. recesp.2014.07.017

20. Mateo J. Nuevos anticoagulantes orales y su papel en la práctica clínica. Revista Española de Cardiología Suplementos. 2013; 13(1 SUPPL.3) 33 - 41. doi: 10.1016/S1131-3587(13)70057-8

21. Hanley C, Kowey P. Are the novel anticoagulants better than warfarin for patients with atrial fibrillation? Journal of Thoracic Disease. 2015; 7(2):165 - 171. doi: 10.3978/j.issn.2072-1439.2015.01.23

22. Heidbuchel $H$, Verhamme $P$, Alings $M$, Antz $M$, Hacke W, Oldgren J, et al. Updated European Heart Rhythm Association Practical Guide on the use of non-Vitamin $\mathrm{K}$ antagonist anticoagulants in patients with non-valvular atrial fibrillation. Europace. 2015; 15(5):625 - 651. doi: 10.1093/europace/euv309

23. Bounameaux H, Camm AJ. Edoxaban: An update on the new oral direct factor Xa inhibitor. Drugs. 2014; 74(11):1209-1231. doi: http://dx.doi.org/10.1007/ s40265-014-0261-1

24. Hapgood G, Butler J, Malan E, Chunilal S, Tran $H$. The effect of dabigatran on the activated partial thromboplastin time and thrombin time as determined by the hemoclot thrombin inhibitor assay in patient plasma samples. Thrombosis and Haemostasis. 2013 ; 110(2):308 - 315. doi: 10.1160/TH13-04-0301

25. Rechenmacher S, Fang, J. Bridging Anticoagulation Primum Non Nocere. Journal of the American College of Cardiology. 2015; 66(12):1392-13403. doi: 10.1016/j.jacc.2015.08.002

26. Díaz J, Duque M, Duque L, Uribe W, Medina E, Marín J. Tratamiento perioperatorio del paciente con antiagregación o anticoagulación. Revista Colombiana de Cardiología. 2012; 19(5): 252 - 259. doi: 10.1016/ S0120-5633(12)70141-9

27. Lai A, Davidson N, Galloway S, Thachil J. Perioperative management of patients on new oral anticoagulants. The British journal of surgery. 2014; 101(7): 742 - 9. doi: 10.1002/bjs.9485

28. Skaistis J, Tagami T. Risk of fatal bleeding in episodes of major bleeding with new oral anticoagulants and Vitamin K antagonists: A systematic review and me- 
ta-Analysis. PLoS ONE. 2015; 10(9): e0137444. doi: 10.1371/journal.pone. 0137444

29. Ansell J. Reversal Agents for the Direct Oral Anticoagulants. Hematology/Oncology Clinics of North America. 2016; 30(5): 1085-1098. doi: 10.1016/j. hoc.2016.05.006

30. Siegal DM, Curnutte JT, Connolly SJ, Lu G, Conley PB, Wiens BL, et al. Andexanet Alfa for the Reversal of Factor Xa Inhibitor Activity. N Engl J Med. 2015; 373(25):2413-2424. doi: 10.1056/NEJMoa1510991

31. Pollack CV, Reilly PA, Eikelboom J, Glund S, Verhamme $\mathrm{P}$, Bernstein RA, et al. Idarucizumab for Dabigatran Reversal. NEnglJMed. 2015; 373(6):511 - 520. doi: 10.1056/NEJMoa1502000
32. Ansell JE, Bakhru SH, Laulicht BE, Steiner SS, Grosso M, Brown K, et al. Use of PER977 to Reverse the Anticoagulant Effect of Edoxaban. New England Journal of Medicine. 2014; 371(22):2141 - 2142. doi: 10.1056/NEJMc1411800

33. Connolly SJ, Crowther M, Eikelboom JW, Gibson CM, Curnutte JT, Lawrence JH, et al. Full Study Report of Andexanet Alfa for Bleeding Associated with Factor Xa Inhibitors. N Engl J Med. 2019; 380(14):1326 - 1335. doi: 10.1056/NEJMoa1814051. 